\title{
Enhancing the QoS in Hybrid Wireless Networks
}

\author{
Kishori Dharurkar ${ }^{1}$, Dipak Patil ${ }^{2}$ \\ ${ }^{1}$ Information Technology, Pune University, Amrutvahini College of Engineering, Sangamner, India \\ ${ }^{2}$ Information Technology, Pune University, Amrutvahini College of Engineering, Sangamner ,India
}

\begin{abstract}
This paper proposes a distributed Quality of Service (QoS) oriented routing algorithm for hybrid wireless network. The hybrid wireless network is combining advantages of infrastructure networks and mobile ad hoc networks (MANET). The wireless applications are deploying day by day very rapidly. These applications are demanding robust networks with end to end QoS requirements. Hybrid wireless networks will be the best next generation network. So providing QoS routing algorithm for such networks it on demand research. Here the QoS-Oriented Distributed routing protocol (QOD) is proposed to enhance the QoS support of hybrid wireless networks. This protocol consists of 6 algorithm 1.Neighbor selection algorithm, 2.Malicious Node Detection 3.Packet distribution scheduling 4. Node mobility speed based packet assigning, 5. Packet data compression deflate algorithm 6.Traffic redundancy elimination algorithm. This protocol improves the QoS of hybrid wireless networks. The simulation results show that packet delay, packet loss is getting improved than existing system.
\end{abstract}

Keywords: Hybrid wireless networks, MANETs, QoS, Routing

\section{Introduction}

The wireless environment is increasing very rapidly. The internet users are also getting added in millions yearly. With this growth the multi interface mobile devices (e.g., smartphone and tablet PC) are growing. People like to be online, live online. So number of multimedia applications is getting deployed every day. In such a dynamic environment the multimedia services like online movies, online TV, mobile gaming, and online conferences etc., need the robust end to end network support. This motivated the need of high Quality of Service (QoS) support in wireless and mobile networking environments .The QoS support reduces end- toend transmission delay. It provides throughput to guarantee the robust communication between mobile devices and wireless infrastructures.

Hybrid wireless networks take the advantage of existing infrastructure networks and also utilize the MANET properties in absence of infrastructure. Hybrid wireless networks provide tradeoff between cellular networks and MANETs. Hybrid networks are the next generation networks they can meet quality requirements.

The proposed algorithm is two hop routing algorithm. In two hop forwarding basically the source node send packet directly to A.P. if it is in range of it. Otherwise it use relay node for forwarding it to A.P. Hybrid wireless network has the feature of any cast transmission and number of hops between mobile node and access point are small. Due to any cast transmission the access point can be a source or destination to any mobile node. The small hop gives us to connection to A.P. through relay node. This algorithm provides the steps for selecting the intermediate relay node then assigning the packet load to them depending upon their scheduling feasibility. It also considers the nodes mobility speed, redundant data packet while forwarding. Scheduling feasibility is the ability of node for forwarding the data with QoS.

The below figure 1 shows architecture of hybrid wireless network.

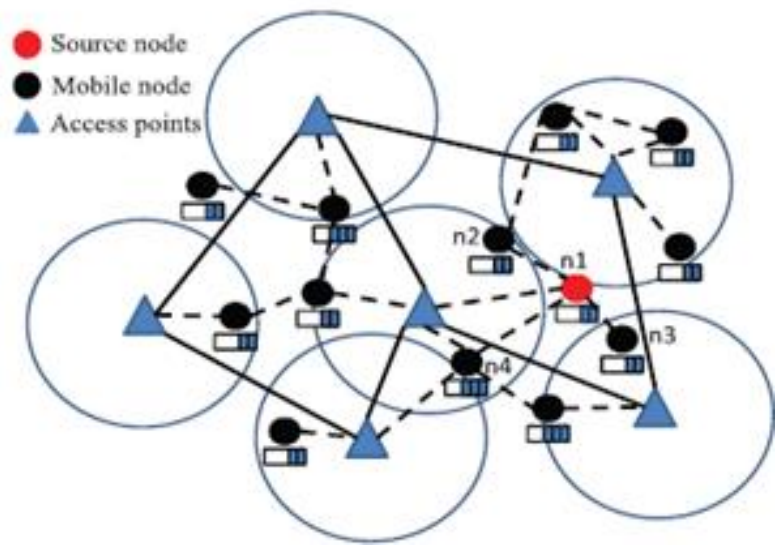

Figure 1: Hybrid Wireless Networks

The below algorithm is shows pseudo code for distributed routing algorithm.

if receive a packet forwarding request from a source node then

if this Space Utility $<$ threshold

then

Reply to the source node.

end if

end if

if receive forwarding request replies for neighbor nodes then

Check for the malicious node and remove it from list.

Determine the packet size to each neighbor i

Estimate the queuing delay for the packet for each neighbor. Determine the qualified neighbors that can satisfy the deadline requirements based on.

Sort the qualified nodes in descending order of . Allocate workload rate Ai for each node. For each intermediate node in the sorted list do Compress the packets before sending.

Check for duplicate packet eliminate redundant data by TRE Send packets to with transmission interval end for 
end if.

\begin{tabular}{|c|c|}
\hline Symbol & Indication \\
\hline $\mathrm{N}$ & \# of network nodes \\
\hline$n_{i}$ & Node i \\
\hline$U_{s}$ & Space utility \\
\hline$W_{i}$ & Bandwidth of $n_{i}$ \\
\hline$T_{v}$ & Transmission time of packet p \\
\hline$T_{a}$ & Packet arrival time \\
\hline$T_{a s s}$ & Delay QoS requirement \\
\hline$T_{w}$ & Queuing Delay \\
\hline$N_{q}$ & Set of nodes satisfying deadline \\
\hline$U_{a s}(\mathbf{i})$ & Available space utility \\
\hline$T_{v_{x}}$ & Space utility threshold \\
\hline
\end{tabular}

This routing algorithm comprises of 6 algorithms:

\section{Neighbor Selection Algorithm}

This algorithm first selects the qualified neighbors such that they should provide QoS guarantee. Then by using Earliest Deadline First (EDF) algorithm the packets are forwarded for scheduling further. [1]

\section{Malicious Node Detection}

This algorithm is for detecting misbehavior node so that packet drop should not happen. Here the AODV (Ad hoc On-Demand Distance Vector) algorithm is used for route request and route reply. If route reply is not coming within some set time it is considered as malicious node. [2]

\section{Packet distribution scheduling}

This algorithm then schedules the selected packets depending upon packet deadline. Here the packet transmission time is also considered. Here the value for time with QoS is calculated and packets are scheduled accordingly. [3]

\section{Node mobility speed based packet assigning}

Here the mobility speed of mobile nodes is taken into consideration. The small size packets are given to nodes having high speed. And large size packets are assigned to less mobile nodes. Here packet transmission time is minimized. [3]

\section{Packet data compression}

This algorithm checks for the duplicate strings, letters present in packet data. The deflate compression algorithm is used for compression. Here the packet size gets reduced. Hence the packet delay, bandwidth consumption can be minimized.

\section{Traffic redundancy elimination algorithm}

Here before forwarding the packets, the nodes checks that whether this packet is already send in network or not. If it is present then it only send its signature instead of whole packet data sending thereby reducing network traffic. Here network overload get minimized. [3]

\section{Proposed System}

The distributed QoS oriented routing algorithm consists of 6 algorithms.

\subsection{Neighbor Selection Algorithm}

This algorithm gives us the guaranteed intermediate neighbor nodes. The space utility of node is the fraction of time a node is busy with packet forwarding over a unit time. This algorithm uses the concept of queue length threshold in random early detection (RED) algorithm [12] for setting the space utility threshold. The node sets a threshold depending upon the bandwidth allocated to it. It accept load less than threshold so that node should not be overloaded to provide QoS. When the source node sends the request message to its neighbor nodes the eligible nodes only reply. Along with reply it sends its available workload, packets already in queue, size of those packets. Depending upon the queue condition the source node selects the qualified nodes for forwarding the packets. The packets are given priorities depending upon its deadline. The earliest deadline scheduling first (EDF) algorithm is used for priority assigning.[1]

\subsection{Malicious Node Detection Algorithm}

After selecting the qualified neighbors the source node checks for malicious node. It uses the AODV ad hoc on demand distance vector routing algorithm for sending route request RREQ to check malicious node. If the reply RREP is not coming within some set time then it is declared as malicious. And this node is removed from list. This algorithm avoids the packet drop, packet delay due to malicious nodes.[2]

\subsection{Packet Scheduling Algorithm}

Here the packets are scheduled by considering its deadline. Then the selected packets and selected nodes are scheduled accordingly. The packet transmission time i.e. the time for transmission from source node to intermediate node and from intermediate node to destination node , arriving time , packet deadline time $\mathrm{d}$, packet queuing time of node are calculated. The time is set for checking time requirements of packet within deadline. Then nodes which satisfies this condition are then selected for scheduling.[3]

\subsection{Mobility based Packet Resizing}

Here the large sized packets are given to less mobile nodes and the small sized packets are given to the nodes with high speed. This algorithm utilizes the mobility speed of nodes.[3]

\subsection{Traffic Redundancy Elimination Algorithm}

In network due to broadcasting the huge traffic overload is present. Here we check for the duplicate packet chunks by signature matching. The chunks that are already send in network, the only signature is send. Here the signature is obtained by using SHA-1 algorithm.

\subsection{Deflate Compression Algorithm}

This algorithm is to eliminate redundant data from packet. The contents of packets are checked for duplication and then 


\section{International Journal of Science and Research (IJSR) \\ ISSN (Online): 2319-7064}

Index Copernicus Value (2013): 6.14 | Impact Factor (2014): 5.611

compressed. Here we use deflate lossless compression algorithm. This algorithm minimizes the packet size.[13].

\section{Results}

This project is simulated by using socket programming. The implementation shows that after applying the routing algorithm the packet loss is minimized. The malicious node detection eliminates the malicious node so the chances of packet loss at malicious node are reduced. The compression of packet data reduces the packet size. Hence QoS of routing algorithm is improved. The packet delivery rate and packet loss, packet delay are improved. The snapshots shows the results.
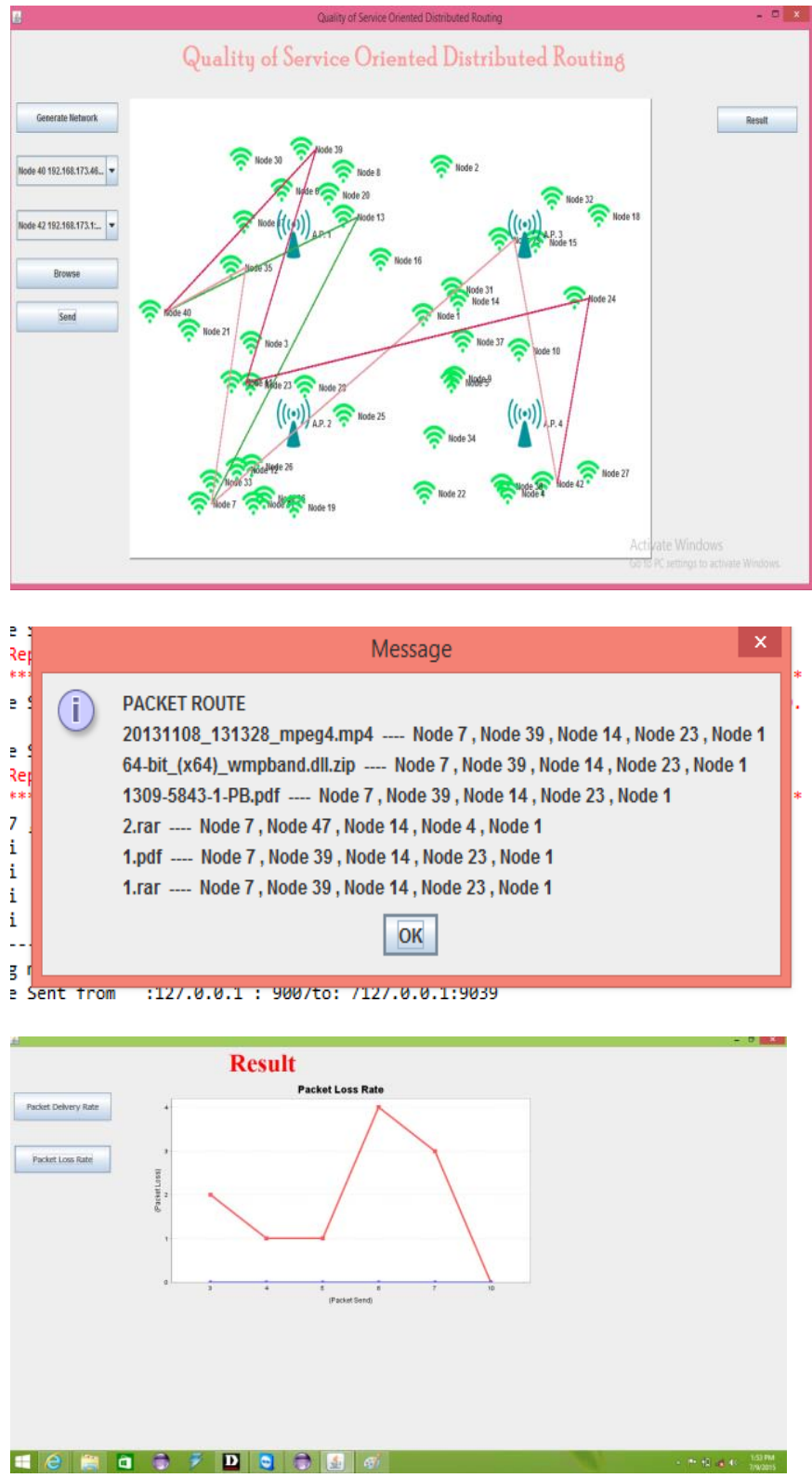

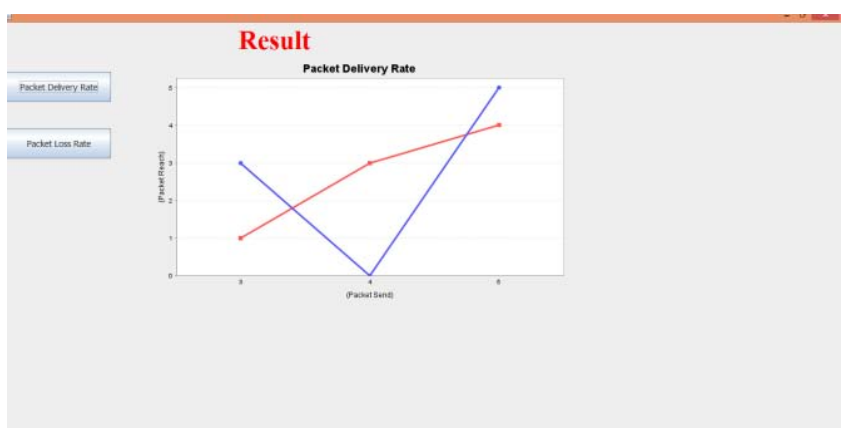

\section{References}

[1] C. Liu and J. Layland, "Scheduling Algorithms for Multiprogramming in a Hard Real-Time Environment," J. ACM vol. 20, pp. 46- 61, 1973.

[2] C.E. Perkins, E.M. Royer, and S.R. Das, Quality of Service in Ad Hoc On-Demand Distance Vector Routing, IETF Internet draft, 2001.

[3] Ze Li, Haiying Shen," A QoS-Oriented Distributed Routing Protocol for Hybrid Wireless Networks" IEEE Trans. Mobile Comp. Vol. 13, No. 3, 2014

[4] R. Braden, D. Clark, and S. Shenker, Integrated Services in the Internet Architecture: An Overview, IETF RFC 1633, 1994.

[5] E. Crawley, R. Nair, B. Rajagopalan, and H. Sandick, Resource Reservation Protocol RSVP, IETF RFC 2205, 1998.

[6] J. Kurose and K. Ross, Computer Networking: A TopDown Approach Featuring the Internet. Addison Wesley, 2004.

[7] I. Jawhar and J. Wu, "Quality of Service Routing in Mobile Ad Hoc Networks," Network Theory and Applications, Springer, 2004.

[8] S. Ibrahim, K. Sadek, W. Su, and R. Liu, "Cooperative Communications with Relay-Selection: When to Cooperate and Whom to Cooperate With?" IEEE Trans. Wireless Comm., vol. 7, no. 7, pp. 2814-2827, 2008.

[9] A. Bletsas, A. Khisti, D.P. Reed, and A. Lippman, “A Simple Cooperative Diversity Method Based on Network Path Selection," IEEE J. Selected Areas in Comm., vol. 24, no. 3, pp. 659-672,2006.

[10] T. Ng and W. Yu, "Joint Optimization of Relay Strategies and Resource Allocations in Cellular Networks," IEEE J. Selected Areas in Comm., vol. 25, no. 2, pp. 328-339, 2004.

[11] J. Cai, X. Shen, J.W. Mark, and A.S. Alfa, "SemiDistributed User Relaying Algorithm for Amplify-andForward Wireless Relay Networks," IEEE Trans. Wireless Comm., vol. 7, no. 4, pp. 1348-1357, 2008.

[12]D. Lin and R. Morris, "Dynamics of Random Early Detection," Proc. ACM Special Interest Group Data Comm. (SIGCOMM), 1997.

[13] T.Arumuga ,Maria Devi “A High Compression Deflate Algorithm for Video Stream" IJCSNS International Journal of Computer Science and Network Security, VOL.12 No.8, 2012 